\title{
Editorial
}

\section{Function Spaces and Operators with Applications}

\author{
Yongqiang Fu, ${ }^{1}$ Qingying $\mathrm{Bu}^{2}$ Donghai $\mathrm{Ji}^{3}$ and Lars Diening ${ }^{4}$ \\ ${ }^{1}$ Department of Mathematics, Harbin Institute of Technology, Harbin 150001, China \\ ${ }^{2}$ Department of Mathematics, University of Mississippi, Oxford, MS 38677, USA \\ ${ }^{3}$ Department of Mathematics, Harbin University of Science and Technology, Harbin 150080, China \\ ${ }^{4}$ Mathematisches Institut der Universität München, Theresienstraße, 80333 München, Germany \\ Correspondence should be addressed to Yongqiang Fu; fuyongqiang@hit.edu.cn
}

Received 29 May 2014; Accepted 29 May 2014; Published 22 June 2014

Copyright (c) 2014 Yongqiang Fu et al. This is an open access article distributed under the Creative Commons Attribution License, which permits unrestricted use, distribution, and reproduction in any medium, provided the original work is properly cited.

The theory of function spaces and operators along with their various applications is an active branch of mathematics and there were a large number of papers published in the relative areas and many major discoveries have been obtained. We are happy that this special issue has received the attention of many researchers in these areas.

The special issue consisted of twelve research papers covering commutators of multiliner Fourier multiplier operators on weighted Lebesgue spaces, fractional Hardy operators and their commutators on Hardy spaces, Marcinkiewicz integrals in weighted weak Hardy spaces, geometry of normed linear spaces (including Banach spaces and Hilbert spaces), ArzelaAscoli theorem for demilinear mappings in topological linear spaces, Malliavin derivatives in variable exponent spaces, the theory of Young measures in variable exponent Sobolev spaces and applications, the applications of reproducing kernel spaces on binary singular problems and nonlinear fourth-order differential equations with integral boundary conditions, and weighted Lorentz spaces and operators.

We hope that readers interested in functional analysis will find useful information in this special issue. At the same time we also hope that in the near future we can see new papers to be published based on this special issue.

\section{Acknowledgment}

Each paper has been peer-reviewed and the editors thank all the contributors and colleagues who spared time and were prompt in refereeing work. 


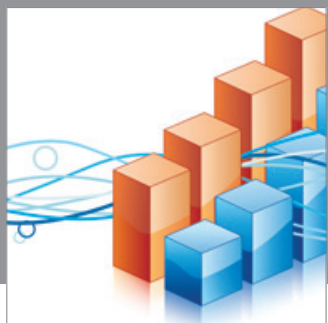

Advances in

Operations Research

mansans

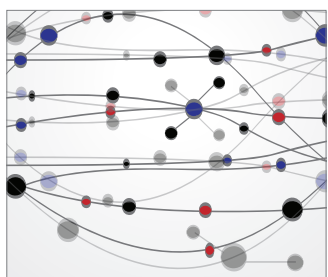

The Scientific World Journal
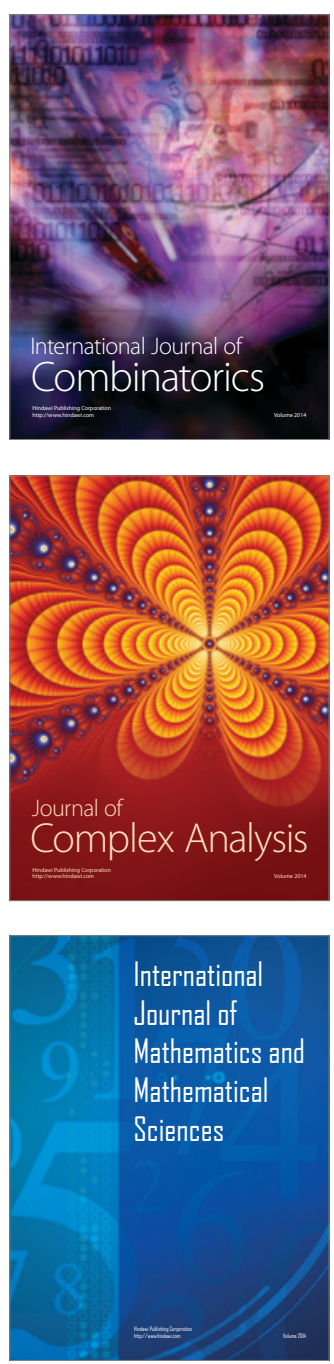
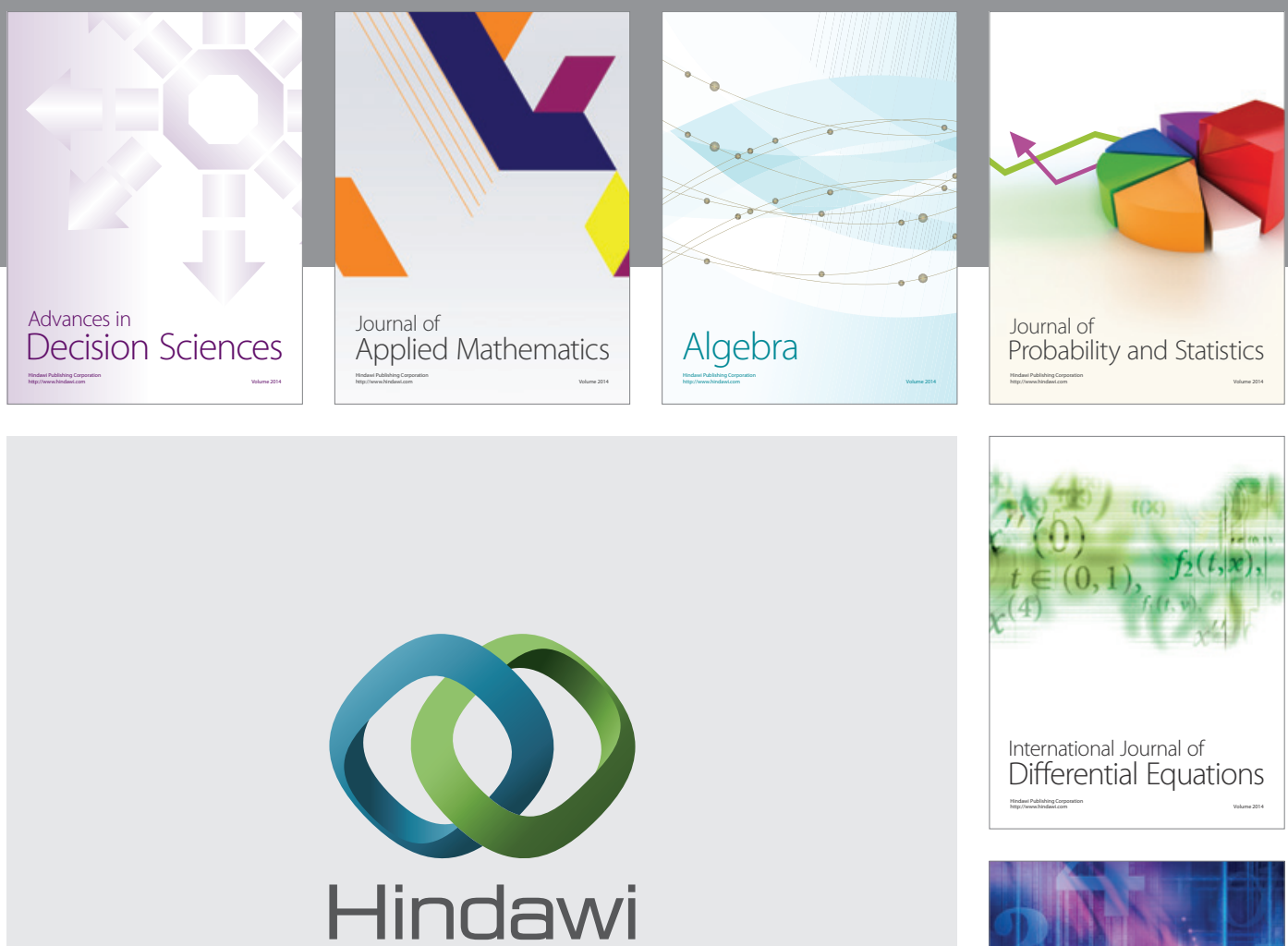

Submit your manuscripts at http://www.hindawi.com
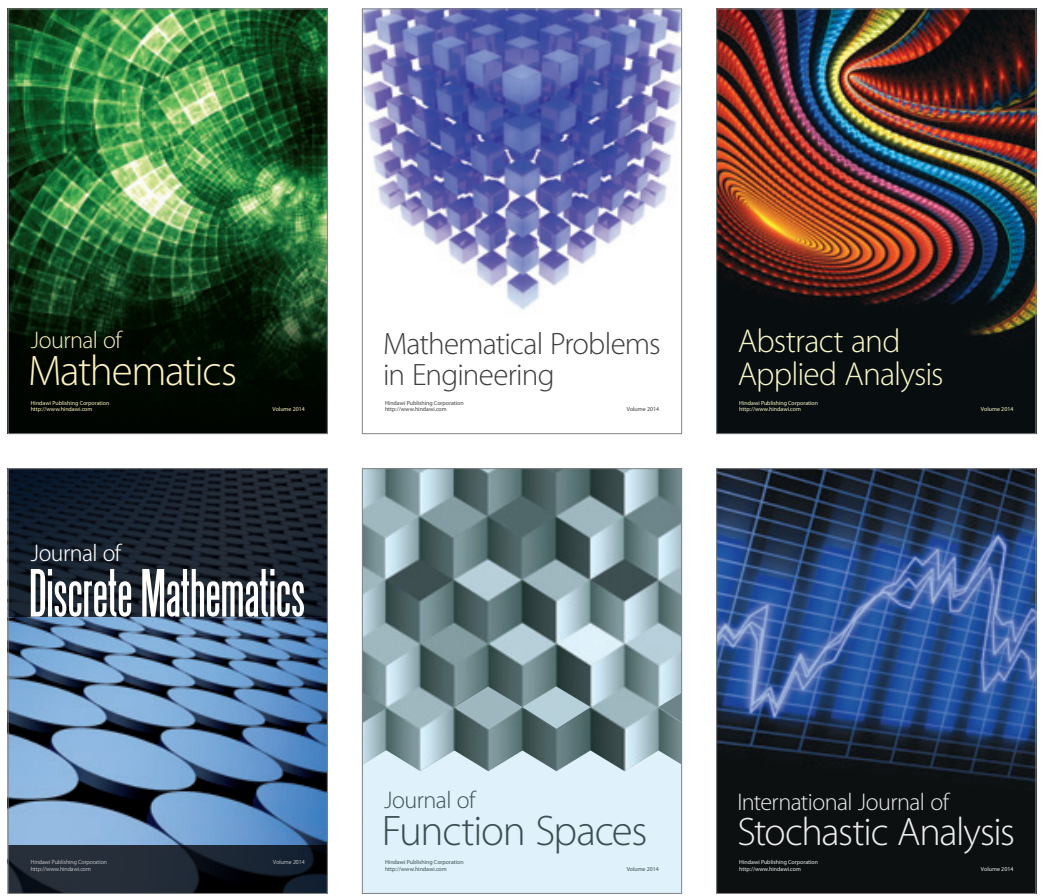

Journal of

Function Spaces

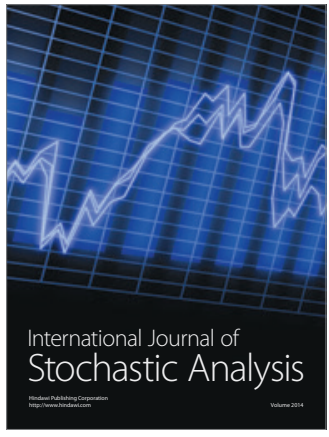

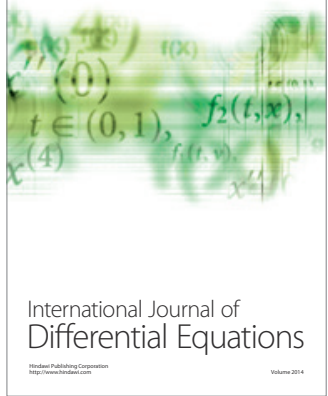
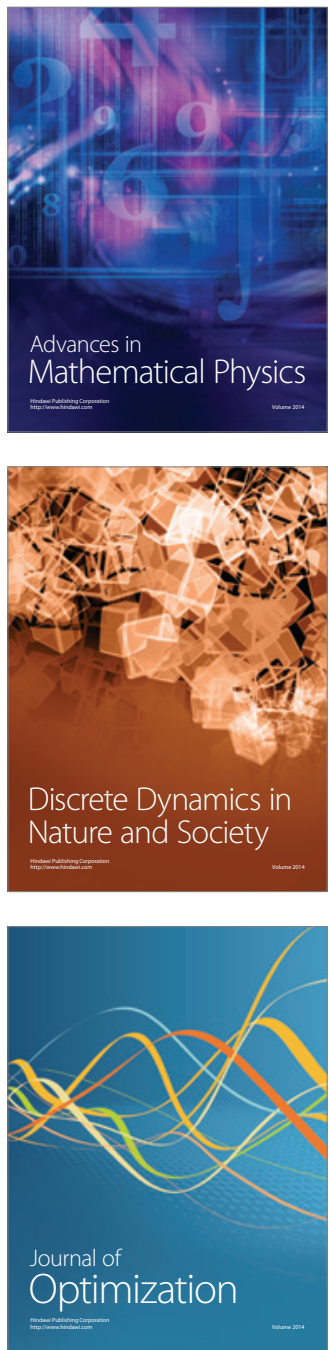